\title{
Quelques mots sur les auteurs
}

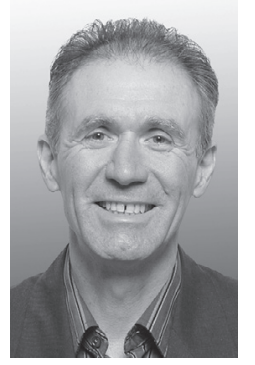

Michel Lequime est Professeur d'Optique à l'École Centrale Marseille et responsable de l'équipe Couches Minces Optiques de l'Institut Fresnel.

Michel LEQUIME est titulaire d'un diplôme d'Ingénieur de l'Institut d'Optique Graduate School (1974), d'un DEA de Physique atomique et moléculaire (1975) et d'un Doctorat de l'université Paris-Sud (Processus optiques du $3^{e}$ ordre en régime picoseconde, 1977), ainsi que d'une Habilitation à Diriger des Recherches délivrée par l'université d'Aix-Marseille (Application de l'interférométrie en lumière blanche à la réalisation de capteurs à fibres optiques à usage industriel, 1996).

Après avoir débuté sa carrière comme Attaché de recherche au CNRS (Laboratoire d'optique quantique de l'École Polytechnique), il a rejoint en 1979 la société de recherche sous contrat BERTIN \& Cie, où il a participé à la création et au 
développement d'une activité centrée sur l'optique et l'optoélectronique. Les projets dans lesquels il s'est impliqué ont majoritairement concerné le domaine des capteurs à fibres optiques (centrale de mesure pour réseau de capteurs à codage de modulation spectrale, système dédié à la mesure de la pression et de la température en fond de puits de pétrole) et celui de l'optique spatiale (télescope de la caméra HMC équipant la sonde européenne Giotto, Balise à fibres optiques de l'expérience de télécommunications optiques intersatellites SILEX).

En 1998, il a quitté BERTIN et participé à la création d'une start-up, Light Technologies, qui visait à développer les applications industrielles des diodes électroluminescentes de puissance qui commençaient alors à être commercialement disponibles (source bleue de polymérisation en bouche de composites dentaires, système de contrôle en ligne de la coloration de produits pétroliers).

En 2000, il a été recruté par l'École Nationale Supérieure de Physique de Marseille (aujourd'hui devenue Centrale Marseille) comme Professeur en Optique et en Gestion de projet, et il a ensuite pris, en 2002, la responsabilité de l'équipe RCMO (Recherche en Couches Minces Optiques) de l'Institut Fresnel.

Les travaux actuels de Michel LEQUIME portent principalement sur la modélisation des propriétés spectrales des cavités planaires multicouches, sur la réalisation de composants de filtrage à forte structuration spatiale, et sur le développement de montages permettant l'enregistrement des propriétés angulaires, spatiales, spectrales et polarimétriques de la lumière diffusée par des surfaces ou des milieux stratifiés.

Il est auteur de plus de 40 publications dans des revues internationales à comité de lecture et de 140 communications dans des conférences nationales et internationales. Il a en outre occupé le poste de Président du Comité Stratégique du pôle OPTITEC de 2006 à 2008 et celui de Secrétaire de la Société Française d'Optique de 2009 à 2013.

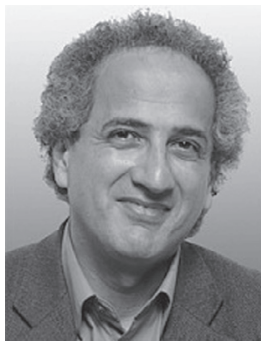

Claude Amra est Directeur de Recherche au CNRS et responsable de l'équipe CONCEPT de l'Institut Fresnel.

Claude AMRA intègre le CNRS en 1986 après une thèse portant sur la diffusion de la lumière par les surfaces des filtres interférentiels multicouches (Laboratoire d'Optique des Surfaces et des Couches Minces, ESA CNRS, École Nationale Supérieure de Physique de Marseille). Ses travaux l'amènent à étendre la modélisation électromagnétique au cas des volumes hétérogènes puis aux microcavités luminescentes, 
pour l'optique en espace libre et pour l'optique modale. Parallèlement il se forme à la métrologie optique et adresse les problèmes inverses inhérents à la caractérisation des milieux désordonnés, avant de s'impliquer dans la technologie des couches minces optiques.

Après une Habilitation à Diriger des Recherches soutenue en 1991 à l'université d'Aix-Marseille, il initie ou contribue activement à l'introduction de nouveaux thèmes au LOSCM : endommagement laser et thermique photo-induite, microscopie à force atomique et rugosité multi-échelle, lumière piégée, microcavités, ellipsométrie de speckle, absorbeurs de lumière achromatiques en couches minces, éclateurs spectraux pour le moyen infra-rouge, multiplexage dense en longueur d'onde, technologies de dépôt par pulvérisation de faisceau d'ions...

En 1996, Claude AMRA se voit confier la direction du Laboratoire d'Optique des Surfaces et des Couches Minces, qu'il reconstruit thématiquement. En 2000, il est co-créateur de l'Institut Fresnel, une nouvelle unité mixte de recherche (CNRS, AixMarseille université, Centrale Marseille) qui rassemble sur le campus de MarseilleNord l'ensemble des Sciences et technologies de l'optique, l'électromagnétisme et l'image. Au cours de ses 2 mandats de direction (2000-2008), l'Institut Fresnel émergera et s'ancrera sur la scène européenne, avec un bâtiment dédié.

En 2009, il est nommé Directeur adjoint scientifique de l'Institut des Sciences de l'Ingénierie et des Systèmes du CNRS, en appui sur la section 8 du Comité National de la Recherche Scientifique (microélectronique, photonique, électromagnétisme, antennes, énergie électrique et nanotechnologies...). Auparavant il aura présidé ou participé à de nombreux comités auprès de l'ANR, l'AERES, le MESR, le CoNRS, le CNU, la région PACA et les pôles de compétitivité..., en parallèle de l'élaboration de nombreux congrès sur la scène internationale.

Les activités de recherche de Claude AMRA concernent aujourd'hui, dans le cadre de l'équipe CONCEPT qu'il anime à l'Institut Fresnel, l'imagerie en milieu complexe, la polarisation et la cohérence en milieu désordonné, le confinement et l'exaltation optique géante, l'analogie optique/thermique, le couplage optique électromagnétique/optique statistique... 